\title{
Schizophrenia: Current and Perspectives
}

\author{
Melika Ahangar ${ }^{1}$, Ali Mohammad Ahadi ${ }^{1 *}$ and Fatemeh Moradi $^{2}$ \\ ${ }^{1}$ Department of Genetics, Faculty of Science, Shahrekord University, Shahrekord, Iran \\ ${ }^{2}$ Department of genetics, Faculty of Science Islamic Azad University, Flavarjan branch, Flavarjan, Iran
}

*Corresponding author: Ali Mohammad Ahadi, Department of Genetics, Faculty of Science, Shahrekord University, Shahrekord, Iran

\section{ARTICLE INFO}

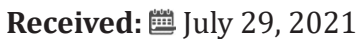

Published: August 06, 2021

Citation: Melika Ahangar, Ali Mohammad Ahadi, Fatemeh Moradi. Schizophrenia: Current and Perspectives. Biomed J Sci \& Tech Res 37(5)-2021. BJSTR. MS.ID.006073.

\begin{abstract}
Schizophrenia is one of the most prevalent nervous system related disorders with a severe and progressive symptom. Main target population of this disease is the people after 60 years old. Several etiological factors are involved in Schizophrenia pathogenesis including trauma, stroke, central nervous system viral infection, genetic and epigenetics elements. We focus here on molecular biology aspect of schizophrenia manifestation. New findings about pathogenesis of this disease and pathways deal with schizophrenia will present in this review and finally we propose here some probable strategies applicable for treatment of this disorder. Molecular and cellular studies of schizophrenia will provide new strategies for management of this complex disorder.
\end{abstract}

Keywords: Schizophrenia; Gene; Pathway; Molecular Biology

\section{Background}

Schizophrenia as a severe and chronic mental disorder which is the most life disruptive disorders in the medicine. There is several types of schizophrenia. people with one type of this disease will develop the other type of disorder with an increased risk [1]. Knowing better about some factors like diet, nutrition and features of metabolic help us for future information in this issue [2]. Some information among these topics is clear to understand scientific benefits people who suffer from this problem. Although information and developments on it are a lot, the latest therapy methods aren't enough completely. Actually, studies about this regard concentrate on the meaning molecular abnormalities [3]. Around $0.7 \%$ of people around the world suffer from this illness. It is true that there are helpful and useful therapies and methods for schizophrenia like antipsychotics. Also, some sick people don't have all symptoms and actually, they have some side effects too [4]. Cognitive deficits and negative symptoms are seen in this illness. In healthy people, neural oscillations and functional networks are connected with a big part of cognitive abilities while in schizophrenia, we can see that neural oscillations are connected with cognitive and negative symptoms in a different range of people. Moreover, symptoms can be seen in different way in sick people [3].
Gaining weight and being fat can be seen in sch. Illness people like to have harmful regimes, higher fat and sweet things and eat fruit and vegetable less than other people [2]. Schizophrenia is a kind of multifactorial disease. there are many factors which are related to biological genes and environmental factors that change and reshape the path of brain development [5]. The base of schizophrenia is involved positive, negative symptoms and cognitive impairment. Positive symptoms have big effects on delusion and hallucination with the ability of speech. Disorganization of speech while negative symptoms are separated in two ways avolition like apathy and diminished expression [6]. Symptoms of heterogeneous consist of hallucination and delusion, avoliation and negative symptoms and cognitive deficits which can cause deficits in working memory, attention and cognitive flexibility [7]. One of the symptoms in schizophrenia is having problem which influence sleeping and working like insomnia, less sleep comfort. Even the rhythm of body temperature can change in this regard [8]. The most visible thing is problematic cognitive which is related to damage in memory, concentration and IQ [9]. There is some evidence about the apparent sickness and emotional procedure in some people who have schizophrenia. People with schizophrenia can have 
different risk for violent actions so they need different strategies during their treatment [10].

\section{Prevalence and Epidemiology}

Schizophrenia generally arises in adolescence. The primary signs that may referred to ultra-high-risk state or psychosis risk syndrome, can be a variety of different symptoms that individuals may present before manifestation of full disease as schizophrenia [11]. Recent research has clarified the changes in risk factor of psychosis like first damaged cognitive and social function. All of the symptoms aren'tstill known, but as far as we concern almost 35\% OF PPL are with first symptoms. Another information about epigenetic shows between fetal hypoxia and hypoxia which is related to genes during childhood trauma and different of serotonin transport and COMT gene on cognitive function [12]. Oxidative stress can increase the risk of this problem, for instance, meta-analysis of studies on oxidative stress markers showed arise levels of the antioxidant red blood cell superoxide dismutase in schizophrenia [1]. Among twins, there is just around 50\% pairwise concordance. bring in that the risk for schizophrenia introduce by polygenic risk scores was 5 times greater than perinatal complications. The macro scale consequences of this can be seen in reductions in grey matter capacity up to adolescence and at first of adulthood [3].

These estimates can be seen during the family environment. Meta-analyses of GWASs for macronutrient intake (such as protein, carbohydrate and fat mass) have explained the connection between eating carbohydrates, fat and protein with the fibroblast growth factor 21 (FGF21) gene and associations of using protein intake with the fat mass and an obesity-associated locus [2]. There is an impractical outcome for these patients is about $40 \%$ and only approximately $16 \%$ can find out their functionality at the social level, and we can pay attention to a reduction of the symptoms. schizophrenia can happen $1 \%$ at the international level with an incidence of 1.5 per 10000 people [6]. At first, we can see that women who suffer this problem is older than men's one. There isn't any visible social factors in this regard. Women's higher level of social development at onset is connected with better mediumterm functional and social results. At first of schizophrenia and depression are the same in length and distinguishable just after positive symptoms.

Plus, Schizophrenia is seen in young males rather than females. All in all, Schizophrenia can occur in all ages and from all walks of life [13]. Studies in Meta-analyses of the data and replication shows schizophrenia risk genes which situated in chromosomes $8 \mathrm{p}$ and $22 q$ also in 1q, 2q, 3p, 3q, 4q, 5q, 6p, 6q, 10p, 10q, 11q, 13q, 14p, $15 q, 16 q, 17 q, 18 q$ and 20q [7]. The reason of changing genetic in schizophrenia and intellectual disability (cognitive deficits) can be in a shared pathobiology [9]. Age is one of the most important factors in this issue. In fact, late youth to early adulthood are important times which schizophrenia can be seen at earlier stages [14]. Schizophrenia is a kind of heterogeneous brain affliction which can be seen firstly at late adolescence or early adulthood' 'Prefrontal cortex is a region that has ways of executive function [15]. While first research showed that prefrontal regions of patients are active more than normal people. Sick people brain studies have shown decreased functional connectivity between the medial prefrontal cortex and ventral striatum during the processing of reward and loss-avoidance Sensory gating deficits in thalamic mal connectivity with the cortex notice us underlie which can have been seen in this illness [10].

Multiple insults to the fetus the risk of schizophrenia in Multiple insults to the fetuscan increaselikematernalstarvation, malnutrition, or smoking, it can be believable that per the neurodevelopmental model of schizophrenia, that maternal infections converge to perturb fetal brain development, so changing implicating altered brain development in a convergent pathophysiology [16]. Cytokines can be necessary in the pathophysiology of SZ. Meta-analyses of cross-sectional studies have shown that antipsychotic naive $\alpha$ [17]. In Patients with a history of serious violence can be seen lower GMV in the right inferior temporal area (the anterior part of the right inferior temporal gyrus expanded to the fusiform gyrus, the middle temporal gyrus, and the temporal pole) and the right [18]. I area (the right insula increases to the ventral diencephalon) than those without a history of violence after controlling for lower years of education and higher positive symptom records [19]. The right inferior temporal area which consists of temporal pole and the central temporal gyrus, and the right insular area were showed in this study as associated with violent behavior. For patients with a history of serious violence. described decrease whole brain and increased putamen sizes [10].

\section{Pathobiology and Etiology}

Recent focus on dimensional approaches and translational biobehavioral research domain criteria maybe helpful to move toward a neuroscience-based definition of schizophrenia. The etiology of schizophrenia is now thought to be multifactorial, with ltiplesmalleffect and fewer large-effect susceptibility genes interacting with several environmental factors [20]. From all these years and treatment response. Schizophrenia is a kind of substantial genetic component, with a high heritability (up to 80\%). There are different environmental factors that can connect with genes to increase the risk of schizophrenia. They focus on the emerging area of investigation which is named epigenetics [21]. Reproducing of risk of psychosis is an interaction between cannabis use and the AKT1 gene. 'Subtle structural alterations, like expanding of the third and lateral ventricles, slight reductions in whole-brain gray 
matter volume, and slight reductions in the volumes of temporal, frontal, and limbic regions can be seen [22]. In etiology, the relationship between multiple genes of small-effect and some rare large-effect genes and unknown environmental factors can be seen. Information which is be helpful about therapeutic interventions move beyond the current sole focus on dopamine toward novel therapeutic targets like glutamate, GABA, and calcium channels [1]

Intervened therapies concentrate on dopamine toward novel therapeutic targets like glutamate, GABA, and calcium channels The Immune response can be involved between schizophrenia and genes. Another factor which affects it is stressful psychosocial, such as life events, and increase of risk of developing schizophrenia [3]. As you know that, poor regime increased body mass index and metabolic disease. Cardiovascular disease is one of first factor contribute to increased mortality is cardiovascular disease (CVD). This effective information shows that eating of fruit and vegetables a lot can be beneficial and has cons, such as decreasing risk for cancer and heart disease [2]. DC2 pathways can be effected by drugs targeting. Hippocampus is one of the brain places which shows in the pathogenesis of schizophrenia. Actually, bulk hippocampus was testes with TWAS [23]. Psychosis can be increased by energy metabolism change. actually, the glucose's oxidative metabolism anomalies. besides, people with schizophrenia have cortical thickness in their brains which can related decrease of IQ [6].

The risk of schizophrenia can be raised during Prenatal infection and increased levels of cytokines in mothers during pregnancy in the offspring.in cortical grey matter, there decrease of volume. moreover, elevated brain cytokines as compared to schizophrenia people without an elevation chronic unresolved inflammation can have big effect on brain vasculature [13]. Stress during life can consist of inflammation in the brain in ill cohort, although levels in peripheral cortisol is decreased in people who have schizophrenia. Also, glucocorticoid receptor mRNA levels can't regulate in the brain of schizophrenia people [24]. The circuit of pre-frontthalamo-cerebellar which disturbs has a big role in the pathophysiology of schizophrenia, and there are many evidence in a decreased capacity of the posterior superior cerebellar vermis. A disturbed prefronto-thalamo-cerebellar circuit has a role in the pathophysiology of schizophrenia [25]. One of the loci, 22q11.2, is already a well -documented risk factor for schizophrenia. On the prefrontal cortex, an area which is associated with cognitive function and shown to be dysfunctional in schizophrenia patients. The metabolic hypo-frontality and prefrontal cortex cell dysfunction that is a big characteristic of schizophrenia, along with a schizophrenia-like pattern of cognitive impairment. Similarly, acute ketamine administration in mice use to illuminate potential early-stage circuit dysfunction in schizophrenia that may then precipitate the long-term neural dysfunction [7].
Schizophrenia has big connection with disrupted cognitive control and sleep-wake cycles. Heterogeneous nature of schizophrenia. Like synapsis II25 and DARPP-3226 have been implicated in disease pathology. Unfortunately, Schizophrenia patients have less life expectancy than other people. Schizophrenia can increase by getting older. Relationship in postmortem brain during whole life and importance of age can have a big effect on gene expression. people who have this problem like to smoke and taking recreational drugs and drinking lots of alcohol which isn't normal as rather of other people [8]. Cognitive deficiency consist of working memory deficits, in sick people with schizophrenia which suggest the consequence of an unstable prefrontal cortical state susceptible to irrelevant distractors and linked to activity in the striatum [14]. Some new research shows that the cerebellum couldn't related with subcortical regions such as the thalamus as well as with cortical regions including the inferior frontal lobe in first-episode schizophrenia patients rather to healthy control subjects [26]. The maternal infection hypothesis of schizophrenia consists of enthusiasm, prompting additional investigation of infectious agents in epidemiological surveys, the Herpes simplex virus, Toxoplasmosis gondi, Coxsackie virus, Cytomegalovirus, and Rubella virus are involved in [16].

White matter abnormalities which are related to disable brain places is a big part in his illness. Neuroprogressive symptoms is a big element in schizophrenia, such as structural brain changes [17]. With schizophrenia as compared to murder without suffering this problem have a decrease of hippocampal and para-hippocampal volume among murderers with schizophrenia. Interestingly, recent studies clarify that, temporal pole and the insula, were showed in individuals with psychopathy or antisocial behavioral problems, as well as reduced thalamic capacity [10]. Actually, schizophrenia is a kind of neurodevelopmental illness with heterogeneous, polygenic, and heritable etiology [27]. Schizophrenia can have a big effect on gross architectural structures, specific cell types, and ion channels among different brain regions, which consists of the prefrontal cortex, thalamus, thalamic reticular nucleus, and basal ganglia. Environmental risk factors and their interactions with gene expression can be important roles in schizophrenia pathophysiology [28].

\section{Cellular and Molecular Biology of Schizophrenia}

We can see different kind of deletion or duplication of sections of DNA (copy number variants) that are connected with arise the risk of schizophrenia on their own but just seen in $2 \%$ to $3 \%$ of people who has schizophrenia. Without any doubt, one of it is a deletion of several mega bases of DNA at chromosome 22q11.2, which increase $30 \%$ to $40 \%$ lifetime risk of schizophrenia. Hypothesis-driven approach investigated the complement 4(C4) locus within the major histocompatibility complex which an 
important part of this issue. Genetic studies with postmortem studies introduce that information about lower levels of synaptic proteins, dendritic spines, and gamma aminobutyric acid (GABA)nergic and glutamatergic markers in patient relative to control participants [3]. There is some information which is shows relationship between SNPs are bi-directional using Mendelian Randomization [2]. Genetic and environmental effects change glycan and lipid PTMs (glycosylation and lipidation, respectively which have a big effect on central nervous system development and its act, which involve in symptom expression and patient treatment response in this multifactorial neuropsychiatric illness. Molecular correlates of neuropsychiatric illness in the context of DNA are seen in schizophrenia (e.g. single nucleotide polymorphism (SNP) expression, genome-wide association studies (GWAS)), RNA (e.g. transcriptomics, in situ hybridization studies), and protein (e.g. expression levels, localization, interaction networks, and functional pathways) [29].

Bipolar diseases and schizophrenia with granule neurons connect with deficiency, while we knew that cell type to sickness have been based on animal models. Also, the extensive genomewide cell-type-enriched eQTL associations, actually some of patient with eQTLs that were not identified in homogenate hippocampus or DLPFC. Two of the most long-standing schizophrenia risk genes which is named GRM3 which showed molecular evidence using this DG-GCL dataset, rather than merely identifying variants proximal to these genes. finally, a powerful balance between unbiased singlenucleus and homogenate tissue sequencing shows cell populations in postmortem human brain provides a between unbiased singlenucleus and homogenate tissue sequencing that can identify cellular and spatial associations with common molecular and clinical traits [23]. There are anomalies in the thickness of cortical grey matter, actually, the almost entire cortex is affected (the primary visual cortex did not show any modifications). Also, the brain structural abnormalities and the hippocampal volume deficits, higher rates of reduction of the hippocampus, ventricles, thalamus, and amygdala and nucleus accumbens can be seen together with significant structural abnormalities [6].

Antagonists which increase by receptors of desensitizing which has beneficial for a long time .otherwise, one of new psychiatry can develop new molecules which have big influence on dopamine neurons, which is seen in the dorsolateral prefrontal cortex, but also on hippocampal neurons. When mitochondrial doesn't work well can cause many problems like chromosome 6 in schizophrenia and chromosomes 18 and 21 in bipolar can be problematic. Genes in the immunological and neuronal paths connected with the schizophrenia grew in the childhood and was completely present in adolescence Elevated pro-inflammatory cytokines are in both blood and brain of people with schizophrenia. Elevated immune-related transcripts including IL-1 $\beta$, IL- 6 and IL-8 in the prefrontal cortex in schizophrenia. Peripheral measures of soluble ICAM1 (sICAM1) and VCAM1 have been reported to be both decreased and increased in schizophrenia dependent on the stage of illness and medication status. Breakdown of transport function and barrier function in schizophrenia van alter in gene expression [24].

Polymorphisms in the OXT and OXTR genes can cause in schizophrenia like Cortical brain regions like the prefrontal and superior temporal cortex and the cerebellum were linked to regulation of social cognitive [25]. There are some heterogeneity in genetics which are loci and alleles sick people have excess of rare SNVs and idles throughout the genome in genes such as ABCA13 [30]. Histone modifications in post mortem of sick people. Actually, it is one methylation of histone H3-lysine residue in the promoter of GAD1, encoding a GABA synthesizing enzyme [31]. One of the most detected expression DPYSL2.13 genes in people who have scz were correlated with Metabolomic analysis of plasma, 5 aberrant metabolites, including TCF4 and COMT, by constructing a molecular network of metabolites, enzymes, intermediate proteins and schizophrenia genetic risk factors [32]. Positive, negative and cognitive symptoms of schizophrenia can have big effects on Subanesthetic doses of NMDA receptor (NMDA-R) antagonists such as phencyclidine or ketamine. The correlation between AKT1 SNPs and schizophrenia is seen and DISC1 interacts directly with GSK3 $\beta$ [33].

Some genes which connect to oxidative phosphorylation and mitochondrial function can cause rhythmicity in this regard. Actually, rhythmic changes in mitochondrial function can make diurnal differences in neural activity the dIPFC [8]. Seven genes (BCL11B, EP300, EPC2, GATAD2A, KDM3B, RERE, and SATB2), where the associated SNP is located within or close to the gene location. Charge syndrome (CHD7), Rubinstein-Taybi syndrome (EP300) and SATB2- associated syndrome. It is rs6984242 which is evidence of association where the schizophrenia risk allele (G) was associated with lower Verbal IQ. Chromatin modulators of gene expression and that map to loci that are associated with schizophrenia [9]. There are many differences in subtle expression individual genes that change the functioning of various gene pathways in schizophrenia. Postmortem brain studies in past have showed differential gene expression for mitochondrial genes in disease. Gene expression differences in brain can go hand in hand [14]. Also frontal D2 receptor binding potentials have been connected to the manifestation of positive symptoms in schizophrenia. That SGAs would be superior in targeting negative symptoms and cognitive deficits. Dopaminergic hyperactivity within the striatum consider as a core feature of schizophrenia and seems to first steps of illness. Risk alleles of Akt1 and DRD2 genes which influence D2 receptor function had less illness-related reductions in IQ levels with higher doses of antipsychotics [26]. 
Not only There aren't non-random association of polymorphic genetic variants between schizophrenia and rheumatoid arthritis (positive or negative), but also between schizophrenia and inflammatory bowel disease. Research on 19 auto immune diseases like HLA region, shows that a lack of risk allele sharing between schizophrenia and each of the 19 immune diseases. The lack of Correlation between MS and this illness can be shown in GWAS studies. Risk of schizophrenia in individual genome-wide is less. The MHC region can have big factor a structural variant of $\mathrm{C} 4 \mathrm{~A}$ (a gene encoding complement component 4. Increased expression of the $\mathrm{C} 4 \mathrm{~A}$ isotope in brains of patients with schizophrenia. $25 \%$ of genes in the DLPFC transcriptomics suffer a fetal-to-post-natal isoform switch, and that genes within schizophrenia GWAS positive loci are enriched among genes that are dynamically regulated in the PFC across brain development at first [16]. Schizophrenia (SZ) can be considered as a highly heritable psychiatric disorder. The risk of SZ in the general population is $1.0 \%$, but the risk of SZ in firstdegree relatives is as high as $6.5 \%$.

The risk of children developing SZ is approximately 13\% with one affected parent and roughly $46 \%$ with both parents affected. Increasing evidence suggests that individuals at genetic high risk for SZ (GHR-SZ) often share brain structural and immunological abnormalities with patients with SZ .IL-6, a pro-inflammatory cytokine, has a big responsible for the acute phase response in SZ, which leads to the physiologic and biochemical changes during disease states. The role of leptin has been recently considered an adipocyte-derived hormone/cytokine in the nervous system of patients with schizophrenia [17].

FOSB, JUN, and GRIA3 are correlated with other genes in the amphetamine pathway. Next analysis introduced expression of FOS in CNS tissues, FOS which is located in human postmortem dorsolateral prefrontal cortex and brain tissues of a schizophrenia 22q11-deletion mouse model has downregulated. RANBP3L in schizophrenia can be seen in this problem. ACTG2 is connected to schizophrenia. Also, another actin-coding gene, ACTG1is related to brain improvement and haering loss. MMP- mediated ECM can cause to this illness. moreover, MMP modulators can a therapy for healing this schizophrenia [28].

\section{Pathways Involved in Schizophrenia}

Provide an additional explanation to the dopaminergic models for the cognitive symptoms of this illness and may ultimately yield novel pharmacological treatment approaches. Research in Neuropathology notice that schizophrenia is characterized by abnormal maturation of prefrontal networks duringlate adolescence and early adulthood, likely due to excessive pruning of synapses and dendritic spines [34]. Pre- and post-synaptic abnormalities in inhibitory neurons such as the parvalbumininterneuron may disturb these critical neurodevelopmental processes. Recent research using opto-genetics shows that parvalbumin interneurons can a big part in gamma oscillations, which in turn are associated with cognitive function [35]. Myelination (another critical neurodevelopmental process) is involved as abnormal in schizophrenia, as shown by post-mortem studies demonstrating reduced expression of myelin basic protein in cortical regions Disturbances in both myelination and the inhibitory control of synaptic pruning may contribute to cognitive deficits in schizophrenia [36].

These recent advances in neuropathology build upon previous postmortem research demonstrating reduced neuropil, but not a reduced number of neurons, in the brains of adults with schizophrenia some recent work has focused on autoimmune dysfunction as a cause of psychosis. For example, anti-NMDAreceptor encephalitis is a potentially treatable but underdiagnosed cause of psychosis that results from the production of antibodies against NMDA receptors. A number of young individuals presenting with their first episode of psychosis may have detectable autoantibodies against this receptor or other neuronal proteins, such as voltage-gated potassium channels [37]. Moreover, epidemiological data suggest a bi-directional association between psychosis and some common autoimmune diseases [1]. Abnormalities of N-methyl-D-aspartate receptor function and glutamatergic signaling may also consist of to disrupted excitatoryinhibitory balance. modulation in N-methyl-Daspartate receptor function ora7nicotinic receptor signaling could be beneficial in the treatment of negative and cognitive symptoms but replicated evidence of efficacy remains elusive [3]. Deletions at the 15q13.3 locus encompassing CHRNA7 can be affected on schizophrenia. However, there are positive and negative reports of common SNPs in CHRNA7 being associated with schizophrenia. Interest in alpha-7 nAChRs as a therapeutic target predated the genetic evidence that haplo-insufficiency for the CHRNA7 gene may dramatically increase risk for schizophrenia [7].

We can see association of deletion at chromosome $22 \mathrm{q} 11.2$ with 22q11.2 deletion syndrome (22q11.2DS) shows that a clinically relevant can cause of schizophrenia and is one of the strongest known risk factors for schizophrenia. Genetic modifier locus in studies of cardiac phenotypes in 22q11.2DS [38]. Because of striatal D2 antagonism, the first generation of antipsychotics (FGAs) can make extrapyramidal side effects. However, second generation antipsychotics (SGAs) generally have a therapeutic effect at doses with lower striatal D2 receptor blockade. Dysfunctional connectivity of brain signaling pathways is thought to emerge during neurodevelopment Most fMRI connectivity studies use the blood oxygen level dependent (BOLD) signal as a neurovascular index of regional synaptic activity [26]. Positron emission tomography (PET) is one of main neuroimaging modality used to purportedly measure neuro-inflammation in vivo, in patients with chronic or new-onset schizophrenia. Beyond PET 
imaging, magnetic resonance spectroscopy (MRS) has been used as a modality to investigate neuro-inflammation in vivo in patients with schizophrenia. lack of Immune activation, or the up-regulation of immune genes or pathways connect with schizophrenia [16].

These findings suggest that abnormal WM integrity in SZ may reflect the state of disease and is also associated with IL-6 levels. In addition, these leptin-associated WM integrity abnormalities in both SZ and GHR-SZ individuals may reflect the genetic vulnerability to schizophrenia [17]. Lower GMV in the wide area showed significantly lower GMV in the wide area which consist of the right temporal pole, the inferior temporal gyrus, the fusiform gyrus, insula, the ventral diencephalon. Dysfunction of multiple neurotransmitter systems can contribute to the pathophysiology of schizophrenia, including dopamine (DA), glutamate, and serotonin neurotransmission. FOS may have some connection with amphetamine addiction pathway in schizophrenia samples. in schizophrenia, fibroblast and lymphoblast samples regulate up [28].

\section{Interference RNA and Schizophrenia}

Lower messenger RNA levels of parvalbumin and markers of other inhibitory interneuron subtypes, 43 and lower levels of glutamate decarboxylase 67 (GAD67) messenger RNA and GAD67 protein, an enzyme involved in GABA synthesis which the process of them changed. We can consider that microglia are one of the important feature in pruning synapses [3]. We can see Increase of ICAM1 mRNA in schizophrenia. there are some genetics factors like MIR137, four targets of miR-137, CSMD1, a potential suppressor of squamous cell carcinomas, WBP1L, a WW domain binding protein of unknown function, CACNA1C and TCF4. Also, when miRNAs in post-mortem brain can't do its duty can cause sick people to decrease of expression [24]. We can see that different expression between NRG1 and ERBB4 mRNA and protein in post-mortem in brain of schizophrenia patients. NRG1 mRNA is increased in the hippocampus [7]. CMC is one of the biggest brain samples with RNA sequencing data and gene expression differences [14]. In this problem we can see that cytokines-mediated immune can't have enough function [17].

\section{miRNA and Schizophrenia}

The schizophrenia-associated global miRNA expression analysis showed a significant increase in small RNAs. The most considerable up-regulation was in miR-107 and the entire miR-15 family, which may have overlap in the most of their target genes and would be expected to show functionally convergent effect on cortical region of brain gene expression [39].

\section{Drug Therapy}

There are many ways to treat this illness. The results of treatment are different, and many people will be fine after healing.
By introducing of chlorpromazine, antipsychotic drugs are mainly use for treatment of schizophrenia. Well, the most effective SGA for the treatment of refractory schizophrenia, clozapine, is limited by the risk of agranulocytosis by measuring of counting blood cell, we can see that EPSs are lowest with clozapine and highest with haloperidol. All drugs can gain weight more expect haloperidol, ziprasidone and lurasidone, with olanzapine and clozapine can cause gaining weight. There is controversial issue about olanzapine and risperidone over other antipsychotics [40]. Pharmacogenomics is a major that is a growing field in the treatment of schizophrenia and can bring the field of psychiatry closer to achieving evidencebased personalized medicine with the goals of predicting better treatment response and reducing medication-induced side effects.

For instance, polymorphisms in the serotonergic system can be connective with the efficacy of clozapine and risperidone, dopamine D3 receptor polymorphisms are associated with response to clozapine and olanzapine, and D2 variants are associate with the efficacy of risperidone. It can have some side effects too, gaining weight is related to serotonergic system (HTR2C) and hypothalamic leptin-melanocortin genes (MC4R) can predict antipsychotic induced, cytochrome P450 (CYP2D6) and dopamine receptor variants are associated with tardive dyskinesia. Major histocompatibility complex (human leukocyte antigen [HLA]) markers have been consistently found to be associated with clozapine-induced agranulocytosis. "However, Antipsychotic drugs can be helpful but not enough [4]. Many genes with GWAS regions which are connected have common drug targets for different range of drug classes. Dopamine D2-receptor blockers are ways common drug treatments. And studies about positron emission tomograpgy (PET) shows the effect of dopamine D2 receptors, generally more than $60 \%$ occupancy, is required for a high likelihood of response. For these individuals, clozapine can be beneficial, but the mechanism underlying this remains poorly understood.

Lower striatal dopamine synthesis capacity, higher glutamate". "Treatment resistance is connected with gray matter in the anterior cingulate cortex. Many studies support that individual with higher polygenic risk score are less likely to respond to antipsychotic treatment. Also, syudirs show that that individuals with higher polygenic risk score are less likely to respond to antipsychotic treatment [3]. Antipsychotic and antidepressant drugs are related to changes in dentate gyrus gene expression and levels of hippocampal. The goal of many novel therapies is that targeting other neurotransmitter systems such as glycine, glutamate and acetylcholine, as well as second messenger degradation. Several loci. In past, clozapine used to treat schizophrenia while recently show to up-regulate the expression of genes in the MAPK signaling pathway in the mouse brain [23].

Mir $\backslash 137$ can be an effective by targeting synaptic protein synthesis. While clozapine is useful in some ways [7]. Also, our 
results focus on the need for clinical studies with OXTR agonists in schizophrenia [25]. Additionally, we will see many changes in this regard like using additional analytical approaches. For example, non $\backslash 24 \mathrm{~h}$ rhythms or another especial subject covariate can effect expression of rhythmic. Also, finding process by theses changing will be happen which are related to this issue [8]. Actually, there isn't any information about the relationship between this variant and information which aren't dependent. Also, studies in further eQTL, functional and behavioral will increase our knowledge between rs6984242 and CHD7 [9]. Age can be effective in this illness which require early and late disease during lifetime. So, it can cause this illness have different pathology [14].

\section{Post-Translational Protein Modifications in Schizo- phrenia}

Improvements in technologies which access protein PTMs is access glycemic and lipidomic analyses can be accessible in mass spectrometry methods and tools. metabolite and enzyme functional analyses know abnormal lipid species and glycan structures [29]. Genetics, genomic, proteomic and clinical discipline can help us for healing this sickness. Unfortunately, there are millions of sick people who suffer from schizophrenia can these kinds of methods can be effective for them in next days. As you know that there is many evidence which explain that some special genes can related to this problem. However, we need to have more information in this issue. Relationship between macroscoping finding, neural pathways, genetics and prenatal and postnatal like adolescence finding can be effective to decrease of quality and quantity of life in sick people and to access better ways for treatment which need less expanses [6]. Intervention therapies can be focus on glutamate, GABA, and calcium channels. There are some psychotic symptoms which is related to cognitive deficits and negative symptoms [1].

\section{Futures}

Sick people take amisulpride and symptoms which developed while she increases side effects which consist of extrapyramidal which be better after dosage [3]. Actually, depression, emotional health, happiness and life satisfaction in schizophrenia which don't expect it. By the way, consequences between diet which we select and schizophrenia risk which shows that disadvantages in schizophrenia. And factors are related with illness and treatment with drugs. The relations ion between DCs and schizophrenia isn't expected. While it sounds paradoxical results have seen for 2 diabetes (T2D miRNA specially miR-137 is a biomarker which consists of improvements of this problem [6]. Molecular and cellular studies of schizophrenia will provide new strategies for management of this complex disorder.

\section{References}

1. Haller CS, Padmanabhan JL, Lizano P, Torous J, Keshavan M (2014) Recent advances in understanding schizophrenia. F1000prime reports 6: 57.

2. Wong GT, Bonocora RP, Schep AN, Beeler SM, Fong AJL, et al. (2017) Genome-wide transcriptional response to varying RpoS levels in Escherichia coli K-12. Journal of bacteriology 199(7): e00755-e00716.

3. McCutcheon RA, Reis Marques T, Howes OD (2020) Schizophrenia-An Overview. JAMA psychiatry 77(2): 201-210.

4. Ruderfer DM, Charney AW, Readhead B, Kidd BA, Kähler AK, et al. (2016) Polygenic overlap between schizophrenia risk and antipsychotic response: a genomic medicine approach. The Lancet Psychiatry 3(4): 350-357.

5. Zhuo C HW, Li G, Mao F, Li S, Lin X, et al. (2019) The genomics of schizophrenia: Shortcomings and solutions.Prog Neuropsychopharmacol Biol Psychiatry 93(13): 71-76.

6. Trifu SC VA, Trifu AI (2020) Genetic aspects in schizophrenia. Receptoral theories. Metabolic theories. Rom J Morphol Embryol 61(1): 25-32.

7. Winchester CL, Pratt JA, Morris BJ (2014) Risk genes for schizophrenia: translational opportunities for drug discovery. Pharmacology \& therapeutics 143(1): 34-50.

8. Seney ML, Cahill K, Enwright JF, Huo Z, Zong W, et al. (2019) Diurnal rhythms in gene expression in the prefrontal cortex in schizophrenia. Nature communications 10(1): 3355 .

9. Whitton L, Cosgrove D, Clarkson C, Harold D, Kendall K, et al. (2016) Cognitive analysis of schizophrenia risk genes that function as epigenetic regulators of gene expression. Am J Med Genet B Neuropsychiatr Genet 171(8): 1170-1179.

10. Kuroki N, Kashiwagi H, Ota M, Ishikawa M, Kunugi H, et al. (2017) Brain structure differences among male schizophrenic patients with history of serious violent acts: an MRI voxel-based morphometric study. BMC psychiatry 17(1): 105.

11. Gogtay N VN, Testa R, Wood SJ, Pantelis C (2011) Age of onset of schizophrenia: perspectives from structural neuroimaging studies. Schizophr Bull 37(3): 504-513.

12. Barnett JH XK, Heron J, Goldman D, Jones PB (2011) Cognitive effects of genetic variation in monoamine neurotransmitter systems: a population-based study of COMT, MAOA, and 5HTTLPR. Am J Med Genet B Neuropsychiatr Genet 156(2): 158-167.

13. Hafner $H$ (2015) What is schizophrenia? 25 years of research into schizophrenia - the Age Beginning Course Study. World journal of psychiatry 5(2): 167-169.

14. Sabunciyan S (2019) Gene Expression Profiles Associated with Brain Aging are Altered in Schizophrenia. Scientific reports 9(1): 5896.

15. L D Selemon, N Zecevic (2015) Schizophrenia: a tale of two critical periods for prefrontal cortical development. Translational psychiatry 5(8): e623.

16. Birnbaum R, Weinberger DR (2020) A Genetics Perspective on the Role of the (Neuro)Immune System in Schizophrenia. Schizophrenia research 217: 105-113.

17. Wang Y, Wei Y, Edmiston EK, Womer FY, Zhang X, et al. (2020) Altered structural connectivity and cytokine levels in Schizophrenia and Genetic high-risk individuals: Associations with disease states and vulnerability. Schizophrenia research 223: 158-165. 
18. Zhang X LQ, Wang S, Qiu L, Pan N, Kuang W, et al. (2020) Dissociations in cortical thickness and surface area in non-comorbid never-treated patients with social anxiety disorder. EBioMedicine 58: 102910.

19. Kim P TR, Olsavsky AK, Dufford AJ, Erhart A, Hansen M, et al. (2020) Associations between stress exposure and new mothers' brain responses to infant cry sounds. Neuroimage 223: 117360.

20. Howes OD MR, Owen MJ, Murray RM (2017) The Role of Genes, Stress, and Dopamine in the Development of Schizophrenia. Biol Psychiatry 81(1): 9-20.

21. Shorter KR, Brooke H Miller (2015) Epigenetic mechanisms in schizophrenia. Prog Biophys Mol Biol 118(1-2): 1-7.

22. Fatjó-Vilas M SJ, Ibáñez MI, Moya Higueras J, Ortet G, Guardiola Ripoll M, et al. (2020) The effect of the AKT1 gene and cannabis use on cognitive performance in healthy subjects. J Psychopharmacol 34(9): 990-998.

23. Jaffe AE, Hoeppner DJ, Saito T, Blanpain L, Ukaigwe J, et al. (2020) Profiling gene expression in the human dentate gyrus granule cell layer reveals insights into schizophrenia and its genetic risk. Nature neuroscience 23(4): 510-519.

24. Cai HQ, Catts VS, Webster MJ, Galletly C, Liu D, et al. (2020) Increased macrophages and changed brain endothelial cell gene expression in the frontal cortex of people with schizophrenia displaying inflammation. Molecular psychiatry 25(4): 761-775.

25. Uhrig S, Hirth N, Broccoli L, von Wilmsdorff M, Bauer M, et al. (2016) Reduced oxytocin receptor gene expression and binding sites in different brain regions in schizophrenia: A post-mortem study. Schizophrenia research 177(1-3): 59-66.

26. Nejad AB EB, Glenthøj BY, Siebner H (2012) Brain connectivity studies in schizophrenia: unraveling the effects of antipyretics. Curr Neuropharmacol 10(3): 219-230.

27. Herbert Y Meltzer (2017) New Trends in the Treatment of Schizophrenia CNS Neurol Disord Drug Targets 16(8): 900-906.

28. Huang J, Liu F, Wang B, Tang H, Teng Z, et al. (2019) Central and Peripheral Changes in FOS Expression in Schizophrenia Based on Genome-Wide Gene Expression. Frontiers in genetics 10: 232.

\section{ISSN: 2574-1241}

DOI: $10.26717 /$ BJSTR.2021.37.006073

Ali Mohammad Ahadi. Biomed J Sci \& Tech Res

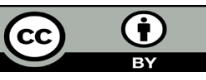

This work is licensed under Creative

Commons Attribution 4.0 License

Submission Link: https://biomedres.us/submit-manuscript.php
29. Mueller TM, Meador Woodruff JH (2020) Post-translational protein modifications in schizophrenia. NPJ schizophrenia 6(1): 5 .

30. Frese FJ, Saks E (2009) Recovery from schizophrenia: with views of psychiatrists, psychologists, and others diagnosed with this disorder. Schizophr Bull 35(2): 370-380.

31. Hsien Sung Huang, Schahram Akbarian (2007) GAD1 mRNA expression and DNA methylation in prefrontal cortex of subjects with schizophrenia. PLoS One 2(8): e809.

32. Edward F Domino, Elliot D Luby (2012) Phencyclidine/schizophrenia: one view toward the past, the other to the future. Schizophr Bull 38(5): 914-919.

33. Effat S Emamian (2012) AKT/GSK3 signaling pathway and schizophrenia. Front Mol Neurosci 5: 33

34. Walker EF TH, Goulding SM, Holtzman CW, Ryan AT, McDonald A, et al. (2013) Developmental mechanisms in the prodrome to psychosis. Dev Psychopathol 25(4 Pt 2): 1585-1600.

35. Lewis DA CA, Glausier JR, Volk DW (2012) Cortical parvalbumin interneurons and cognitive dysfunction in schizophrenia. Trends Neurosci 35(1): 57-67.

36. Flynn SW LD, Mackay AL, Goghari V, Vavasour IM, Whittall KP, et al. (2003) Abnormalities of myelination in schizophrenia detected in vivo with MRI, and post-mortem with analysis of oligodendrocyte proteins. Molecular psychiatry 8(9): 811-820.

37. Kayser MS DJ (2016) Anti-NMDA receptor encephalitis, autoimmunity, and psychosis. Schizophrenia research 176(1): 36-40.

38. Cleynen I, Engchuan W, Hestand MS, Heung T, Holleman AM, et al. (2020) Genetic contributors to risk of schizophrenia in the presence of a 22q11.2 deletion. Molecular psychiatry.

39. Beveridge NJ CM (2012) MicroRNA dysregulation in schizophrenia. Neurobiol Dis 46(2): 263-271.

40. Kar N BS, Chandavarkar R (2016) Clozapine Monitoring in Clinical Practice: Beyond the Mandatory Requirement. Clin Psychopharmacol Neurosci 14(4): 323-329.

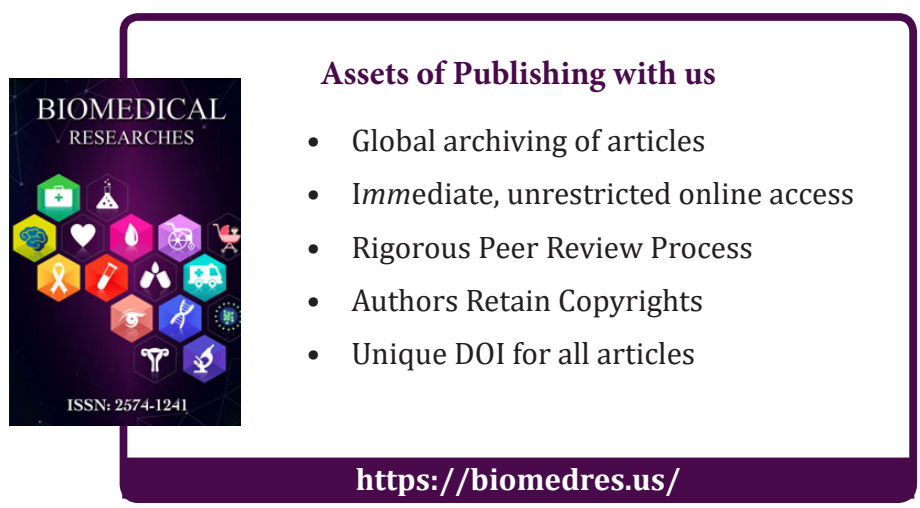

